- Disorders of the blood can affect the timing of dental treatment

- White cell defects influence healing after surgical treatments

- Red cell defects influence healing and the choice of anaesthesia

- Clotting problems impact on surgical treatments and the choice of local anaesthesia

- Anticoagulant medication interacts with drugs used in dentistry

\title{
PRACTICE
}

9

VERIFIABLE

CPD PAPER

\section{General medicine and surgery for dental practitioners Part 9: Haematology and patients with bleeding problems}

\author{
J. G. Meechan ${ }^{1}$ and M. Greenwood ${ }^{2}$
}

Disorders of the blood can affect the management of dental patients. Particular oral signs may be produced. In addition healing may be affected and the choice of anaesthesia for operative procedures will be influenced. Similarly, patients who have problems with haemostasis are a concern. Surgical procedures are obvious problems. However, restorative dentistry is not trouble-free as patients with bleeding problems may present difficulties regarding the choice of local anaesthesia, as regional block techniques may be contraindicated in some patients.

GENERAL MEDICINE AND SURGERY FOR DENTAL PRACTITIONERS:

1. Cardiovascular system

2. Respiratory system

3. Gastrointestinal system

4. Neurological disorders

5. Liver disease

6. The endocrine system

7. Renal disorders

8. Musculoskeletal system

9. Haematology and patients with bleeding problems

10. The paediatric patient

'Senior Lecturer, ${ }^{2}$ Lecturer, Department of Oral and Maxillofacial Surgery, The Dental School, Framlington Place, Newcastle upon Tyne NE2 4BW

Correspondence to: M. Greenwood

E-mail:beryl.leggatt@ncl.ac.uk

\section{Refereed Paper}

doi:10.1038/sj.bdj.4810526

$\odot$ British Dental Journal 2003; 195 :

305-310
Disorders of the blood can be categorised as:

- Problems with red blood cells

- Problems with white blood cells.

Bleeding disorders may be congenital or acquired, for example due to drug therapy. They may be due to:

- Problems with platelets

- Deficiencies in clotting factors

- Vascular problems

- Fibrinolytic problems.

\section{POINTS IN THE HISTORY}

The routine medical history taken from each patient before treatment should include enquiries about the blood and bleeding. Feelings such as lethargy may be produced by many disorders but may indicate an underlying anaemia. Repeated infections may be the result of deficiencies in white cell numbers or function. Regular nose bleeds or bruising after minor trauma should be taken seriously as should episodes of prolonged bleeding after dental extractions. Nevertheless, it is possible that a dental surgical procedure could be the first discovery of a bleeding problem in a patient otherwise thought to be normal. The authors have been involved in a case of a patient in his twenties who had undergone orthopaedic surgery uneventfully but who suffered a life-threatening bleed following the removal of third molar teeth. Investigations performed after that event provided a diagnosis of von Willebrand's disease.

A full drug history should of course be elicited. This is important because, in addition to detecting drugs taken to directly interfere with bleeding, such as warfarin, many drugs can produce bleeding disorders as an unwanted side effect. It is not only prescribed medication that may interfere with haemostasis; drugs of abuse including alcohol and heroin may cause excess bleeding. Finally, any family history of problems with bleeding should be elicited.

When a haematological or bleeding disorder is suspected special investigations to be performed include a full blood count and clotting studies. The ranges of normal haematological values are given in Tables 1 and 2 .

\section{PROBLEMS WITH RED BLOOD CELLS}

Two categories of disorder may occur, these are:

- Anaemia

- Polycythaemia.

\section{Anaemia}

Anaemia is a reduction in the oxygen carrying capacity of the blood. Anaemia may be caused by a number of disease states or because of drug therapy. ${ }^{1}$ This may be the result of reduced numbers of erythrocytes or defects in haemoglobin 


\begin{tabular}{|c|c|c|}
\hline Parameter & Normal range (male) & Normal range (female) \\
\hline Red cell count & $4.5-6.5 \times 10^{12} / \mathrm{L}$ & $3.9-5.6 \times 10^{12} / \mathrm{L}$ \\
\hline White cell count & $4.0-11.0 \times 10^{9} / \mathrm{L}$ & $4.0-11.0 \times 10^{9} / \mathrm{L}$ \\
\hline Platelets & $150.0-400.0 \times 10^{9} / \mathrm{L}$ & $150.0-400.0 \times 10^{9} / \mathrm{L}$ \\
\hline Reticulocytes & $25-100 \times 10^{9} / \mathrm{L}$ & $25-100 \times 10^{9} / \mathrm{L}$ \\
\hline Erythrocyte sedimentation rate & $\begin{array}{c}\text { Upper limit = } \\
\text { age in years } \div 2\end{array}$ & $\begin{array}{c}\text { Upper limit }= \\
\text { (age in years }+10) \div 2\end{array}$ \\
\hline Haematocrit & $0.4-0.54$ & $0.37-0.47$ \\
\hline Haemoglobin & $13.5-18.0 \mathrm{~g} / \mathrm{dL}$ & $11.5-16.0 \mathrm{~g} / \mathrm{dL}$ \\
\hline Mean cell volume & $76-96 \mathrm{fl}$ & $76-96 \mathrm{fl}$ \\
\hline Mean cell haemoglobin & $27-32 \mathrm{pg}$ & $27-32 p g$ \\
\hline Mean cell haemoglobin concentration & $30-36 \mathrm{~g} / \mathrm{dL}$ & $30-36 \mathrm{~g} / \mathrm{dL}$ \\
\hline Red cell folate & $0.36-1.44 \mu \mathrm{mol} / \mathrm{L}$ & $0.36-1.44 \mu \mathrm{mol} / \mathrm{L}$ \\
\hline Vitamin $B_{12}$ & $0.13-0.68 \mathrm{nmol} / \mathrm{L}$ & $0.13-0.68 \mathrm{nmol} / \mathrm{L}$ \\
\hline Prothrombin time & $10-14$ seconds & $10-14$ seconds \\
\hline Activated partial thromboplastin time & $35-45$ seconds & $35-45$ seconds \\
\hline
\end{tabular}

\begin{tabular}{lr}
\hline $\begin{array}{l}\text { Table } 2 \text { Differential white cell } \\
\text { count (normal range) }\end{array}$ \\
\hline Cell & $\%$ \\
\hline Neutrophils & $40-75$ \\
Eosinophils & $1-6$ \\
Basophils & $0-1$ \\
Lymphocytes & $20-45$ \\
Monocytes & $2-10$
\end{tabular}

\section{Oxygen \\ A number of diseases and drugs affect the oxygen carrying capacity of the blood}

function. Red cell numbers can be low because of decreased production due to a deficiency state or bone marrow aplasia. Alternatively, the erythrocyte numbers may be reduced because of increased destruction; this is known as haemolytic anaemia.

Deficiency anaemia can be caused by lack of iron, vitamin $B_{12}$ or folate. The different deficiencies produce different effects on the erythrocyte. Iron deficiency produces small cells, lack of vitamin $B_{12}$ or folate results in large erythrocytes. Deficiency states are corrected by replacement therapy. Iron deficiency may be due to dietary factors or due to loss of blood, for example from an intestinal malignancy. Vitamin $B_{12}$ deficiency, known as pernicious anaemia, is not due to dietary problems but is caused by poor absorption of the vitamin. This is a result of defective intrinsic factor function caused by autoantibody attack. Pernicious anaemia is of interest to dentists as it is one of the complications of nitrous oxide abuse. ${ }^{2}$

Haemolytic anaemia can be the result of extrinsic factors (such as malaria) or problems with haemoglobin. Included among the conditions that produce defects in haemoglobin are sickle cell disease, the thalassaemias and glucose 6-phosphate dehydrogenase deficiency. Sickle cell disease represents a variant in haemoglobin known as haemoglobin S. This haemoglobin causes distortion of the erythrocyte when the oxygen tension is reduced leading to increased haemolysis. Sickle cell disease is a homozygous condition. More common is the heterozygous state - sickle cell trait. This trait is normally asymptomatic and only causes problems when the patient is in a situation of reduced oxygen concentration. Sickle cell disease and trait are more common in patients of African and Afro-Caribbean descent than in other populations.

The thalassaemias may be found in patients of Asian, Mediterranean and Middle-East descent. Like sickle cell disease these conditions increase haemolysis. They are often associated with a cardiomyopathy. The thalassaemias can produce bone-marrow expansion, which can present as maxillary enlargement.

Glucose 6-phosphate dehydrogenase deficiency causes a metabolic disturbance in the erythrocyte leading to an accumulation of oxidants. These oxidants produce methaemoglobin and cause denaturing of haemoglobin with resultant haemolysis.

\section{Polycythaemia}

Polycythaemia is the over-production of red blood cells. It may be a sign of cardiac problems that decrease the amount of blood passing through the lungs, for example a cardiac shunt from the right to the left side of the heart. A serious complication of polycythaemia is thrombosis. The condition may be controlled by frequent bloodletting or by cytotoxic drug therapy.

\section{PROBLEMS WITH WHITE CELLS}

White cell problems can present as:

- Reduced numbers (leucopaenia)

- Increased numbers (leucocytosis)

- Malignancy.

\section{Leucopaenia}

Leucopaenia is a white blood cell count of less than $4.0 \times 10^{9} / \mathrm{L}$. It may be the result of a disease process such as HIV infection or the early stages of leukaemia. Alternatively it may be caused by drug therapy. ${ }^{1}$ Cyclic neutropaenia is a condition in which there are cycles where the white cell count drops. The clinical presentation of leucopaenia is known as agranulocytosis. This produces susceptibility to infection. The dentist may be involved as oral ulceration may occur in this condition.

\section{Leucocytosis}

Leucocytosis is a white cell count of greater than $11 \times 10^{9} / \mathrm{L}$. Many infections raise the white cell count and it is a feature of leukaemia. 


\section{Malignancy}

Malignant diseases of the white cells include:

- Leukaemias

- Lymphomas

- Myeloma.

\section{Leukaemias}

Leukaemias are divided into acute and chronic forms. Two types of acute and two kinds of chronic leukaemia are recognised. These are:

- Acute lymphoblastic leukaemia (ALL)

- Acute myeloblastic leukaemia (AML)

- Chronic lymphocytic leukaemia (CLL)

- Chronic myeloid leukaemia (CML)

Acute lymphoblastic leukaemia (ALL) is the commonest presentation in children. The prognosis in children with ALL is better than that for adults for whom the long-term survival is low.

Acute myeloblastic leukaemia is more common in adults compared to children. The prognosis for both adults and children is poor.

The treatment for the acute leukaemias is with cytotoxic drug therapy or bone marrow transplantation.

The chronic leukaemias involve the proliferation of more mature cells than those found in the acute conditions. The prognosis is better than for the acute leukaemias and adults are more commonly affected than children. Chronic lymphocytic leukaemia (CLL) is the more common form. Some patients with this condition are asymptomatic. The disease may present with splenomegaly (an enlarged spleen) and lymph node enlargement. Chronic myeloid leukaemia (CML) affects adults of a slightly younger age group than CLL. Splenomegaly occurs but lymph node enlargement is not as common as with CLL. Treatment is with chemotherapy and radiotherapy.

\section{Lymphomas}

Lymphomas are divided into two types:

- Hodgkin's

- Non-Hodgkin's

Hodgkin's lymphoma mainly affects males with the peak incidence in the fourth decade of life. It presents as lymph node enlargement. This enlargement often occurs in the neck. NonHodgkin's lymphomas have a poorer prognosis than the Hodgkin's type. Whereas Hodgkin's can be centred on one node non-Hodgkin's is usually multi-focal. Burkitt's lymphoma is a condition associated with the Epstein-Barr virus and may present in the jaws. Treatment for the lymphomas is with combined chemotherapy and radiotherapy.

\section{Myeloma}

Multiple myeloma is a malignancy of plasma cells. It may present in the jaws as a radiolucen- cy associated with loosening of the teeth and altered sensation. Treatment is with chemotherapy. Occasionally an isolated lesion (a plasmacytoma) may occur in the jaws (Fig. 1); these are treated by radiotherapy.

\section{PROBLEMS WITH PLATELETS}

Platelet problems may be:

\section{- Congenital}

- Acquired

The problem may be due to decreased platelet numbers or deficiencies in function. The normal platelet count is over $150 \times 10^{9} / \mathrm{L}$. The lowest level acceptable for dental surgery is $50 \times 10^{9} / \mathrm{L}$. Patients with counts of less than $100 \times 10^{9} / \mathrm{L}$ may show prolonged bleeding but normally local measures such as suturing and the placing

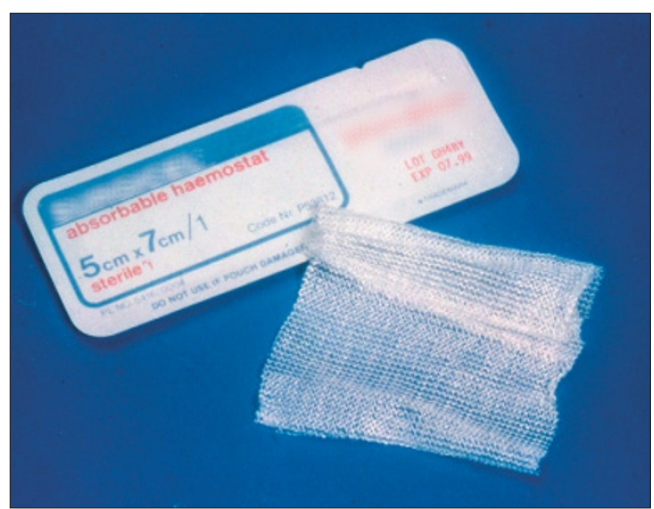

Fig. 2 Resorbable haemostatic agents can be placed in sockets to aid healing

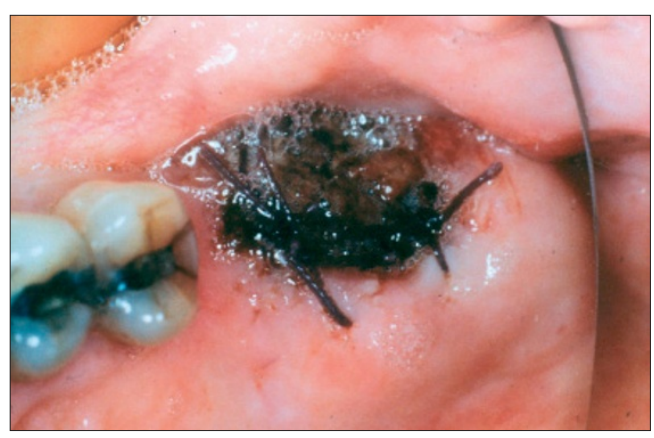

Fig. 3 A haemostatic pack held in place with sutures (courtesy Dr U.J. Moore)

of a haemostatic pack can control this (Figs 2 and 3). Replacement therapy is required if the platelet level is less than $50 \times 10^{9} / \mathrm{L}$. Normally a platelet transfusion will be performed 30 minutes before surgery. If the problem is one of idiopathic thrombocytopaenia oral systemic steroids can be prescribed for 7 to 10 days pre-operatively. This can increase the platelet numbers to a suitable level.

Platelet function can be affected by disease or by drugs. Glanzmann's syndrome is a defect in platelet aggregation. A platelet infusion must be given prior to surgery as bleeding can be severe. A number of drugs interfere with platelet function. Those specifically used for this purpose

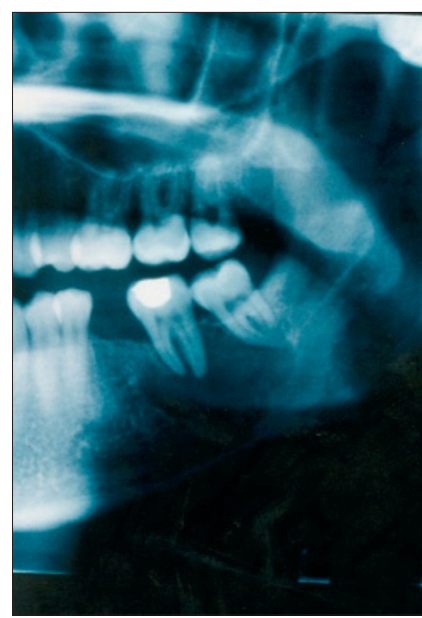

Fig. 1 A plasmacytoma presenting as a mandibular radiolucency at the lower molar apices 
include aspirin and dipyridamole. There is normally no treatment other than local measures required to obtain haemostasis in patients taking these drugs. If there is a concern in a patient taking aspirin then the drug must be stopped for at least 10 days prior to surgery as irreversible changes are produced and replacement with unaffected platelets is needed. Normally, aspirin does not produce problems.

\section{DEFICIENCIES IN CLOTTING FACTORS}

The classic example of a clotting factor deficiency is haemophilia. Haemophilia A is a sex-linked condition that varies in severity. It is due to a deficiency in Factor VIII. Factor VIII function of $25 \%$ or above of normal usually provides satisfactory clotting. Patients with levels of less than 5\% will have symptoms of abnormal bleeding such as easy bruising. When the Factor VIII level is less than $1 \%$ of normal then the condition is classified as severe.

The management of patients with haemophilia who are to undergo surgery relies on a threefold regimen. Therapy can:

- Increase Factor VIII production

- Replace missing Factor VIII

- Inhibit fibrinolysis

Factor VIII levels can be increased by 1-desamino-8-D-arginine vasopressin (DDAVP). In patients with mild forms of the disease this therapy may be sufficient; it may be supplemented with an antifibrinolytic agent (see below) in others.

Replacement therapy is with cryoprecipitate, Factor VIII, fresh frozen plasma or purified forms of Factor VIII. Unfortunately some individuals with haemophilia produce Factor VIII inhibitors. In some cases the level of inhibitors is low and can be combated with high doses of Factor VIII. However, in others the inhibitors are induced in response to Factor VIII and this represents a problem. Inhibitors may be overcome by administering activated Factor IX or prothrombin complex concentrates.

Antifibrinolytic therapy is useful in the postsurgical phase to protect the formed blood clot. Agents used in this way include tranexamic acid and epsilon-amino caproic acid (EACA).

Christmas disease is not as common as haemophilia but is similar to the latter condition with the exception that the problem is reduced Factor IX action. Management is the same as with Haemophilia A except that any replacement therapy is with Factor IX that is not present in cryoprecipitate.

As with haemophilia von Willebrand's disease has variable severity. This condition is not sex-linked and is more common than haemophilia. The disease presents with prolonged bleeding times and reduced factor VIII activity. In mild cases DDAVP and antifibrinolytic therapy are sufficient to cover surgical procedures. However, in severe cases Factor VIII replacement therapy is required.
As well as problems with bleeding these patients may be infected with HIV or hepatitis viruses because of the transfusion of infected blood or blood products.

In addition to congenital causes, problems with clotting may arise due to liver disease or because of drug therapy. An example is the patient who abuses alcohol. Before performing surgery on patients with potential liver problems it is essential to perform a clotting screen to determine if any corrective therapy must be provided to achieve good haemostasis.

Drugs that interfere with clotting include warfarin and heparin. The management of patients on these medications is discussed below.

\section{VASCULAR PROBLEMS}

An example of a vascular disorder is hereditary haemorrhagic telangiectasia. Vascular defects due to deficiency states such as scurvy are rare nowadays, except in some members of immigrant communities. Vascular problems encountered are mainly the result of immunological or connective tissue disease (such as Ehlers-Danlos syndrome). Patients with vascular disorders have a prolonged bleeding time. Local haemostatic measures are all that are usually employed to arrest haemorrhage.

\section{FIBRINOLYTIC PROBLEMS}

Problems with fibrinolysis are not commonly encountered in dental practice. This effect can be produced by drugs (such as streptokinase used as a 'clot-buster' to treat thromboses or pulmonary embolism) or by disease. Plasmin levels may be increased in hepatic or malignant disease such as prostate carcinoma. The treatment is to use an antifibrinolytic agent such as tranexamic acid. This medication is prescribed in hospital practice but is not available for use in the dental practice.

\section{EXAMINATION}

Anaemia may be obvious as pallor of the skin and mucosa but this can be rather subjective. A glossitis may be due to deficiency anaemia. Opportunistic infections or oral ulceration may indicate defects in red or white cells. Gingival enlargement or bleeding, oral paraesthesia or swelling of the parotid glands may be presenting signs of leukaemia. Signs of bleeding disorders may be apparent during the general assessment

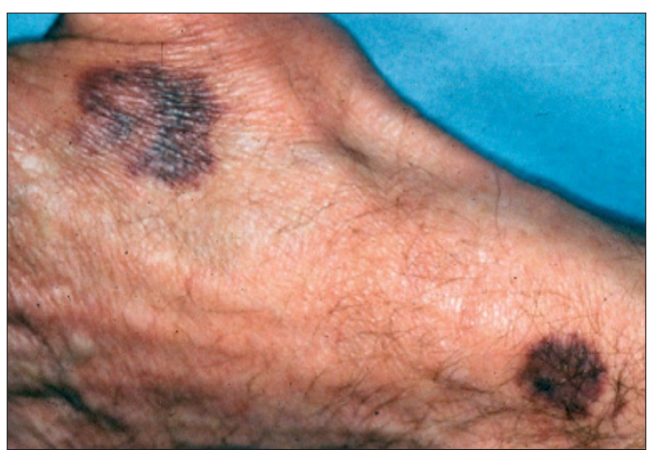

Fig. 4 Bruising on the hand should alert the dentist to a bleeding problem 
of the patient prior to intra-oral examination. Jaundice may be present indicating hepatic disease that can result in clotting problems. Bruising or petechiae on the arms and hands may be visible (Fig. 4). There may also be signs of bruising intra-orally, especially in areas subjected to trauma from the dentition or a denture. Bleeding gingiva in the absence of inflammatory periodontal disease should raise the level of suspicion.

\section{INFLUENCE OF HAEMATOLOGICAL AND BLEEDING DISORDERS ON DENTAL MANAGEMENT}

The management of patients with haematological and bleeding disorders in dentistry may be complex. The following aspects must be considered:

- Surgical procedures

- Choice of anaesthesia

- Medication prescribed

- Cross-infection control

\section{Surgical procedures}

Healing after surgical procedures may be compromised in patients with anaemia or white cell disorders and antibiotics should be administered if this is a concern. The aim is to achieve optimum levels of antibiotic in the forming blood clot so they must be given prophylactically, ${ }^{3}$ not after the procedure. Anaemia is not a contraindication to the provision of minor surgical procedures in dental practice.

The timing of surgery in patients undergoing treatment for conditions such as leukaemia is important. Surgical treatments should be performed during stages of remission and between chemotherapeutic regimens when the cell count is optimal. Close liaison with the supervising haematologist is vital.

No surgical procedure, no matter how minor, should be performed on a patient with a bleeding disorder without prior consultation with the patient's haematologist or physician. Patients with congenital bleeding disorders should be treated in specialist centres where co-operation between surgeon and haematologist is established. Patients with haemophilia A, Christmas disease or von Willebrand's disease may require replacement therapy prior to surgery and an antifibrinolytic agent post-operatively. The use of local measures such as suturing and packing with a haemostatic agent should also be considered to prevent post-operative haemorrhage (Figs 2 and 3).

The management of patients taking drugs that interfere with bleeding is controversial. If aspirin needs to be withdrawn then therapy should cease 10 days before surgery as the effect of the drug on platelets is irreversible and replacement of the platelet population will take this length of time to occur. However, aspirin therapy does not normally need to be stopped and local haemostatic measures normally suffice. Similarly other antiplatelet drugs such as clopidogrel and dipyridamole do not need to be stopped prior to surgery and local measures control bleeding. These patients can undergo minor surgical procedures in general practice.

Patients taking warfarin should have their INR (International Normalised Ratio - a measure of the prothrombin time) measured prior to any surgical procedure. This can now be performed at the chair-side with a finger-prick sample (Fig. 5). The normal therapeutic INR for patients on warfarin is 2.0 to 3.0 except for those with cardiac valve replacement where the range is 2.5-3.5. A level above 4.0 is non-therapeutic and requires adjustment of the warfarin dose. There does not appear to be a universally acknowledged satisfactory INR for dental surgery. One question that has to be addressed is whether it is more dangerous to reduce the level of anticoagulation with the risk of a thrombo-embolic episode than to perform surgery in the warfarinised patient. There is evidence that patients are more likely to die from a thrombo-embolic problem than they are to develop a bleed that does not settle with local haemostatic measures. ${ }^{4}$

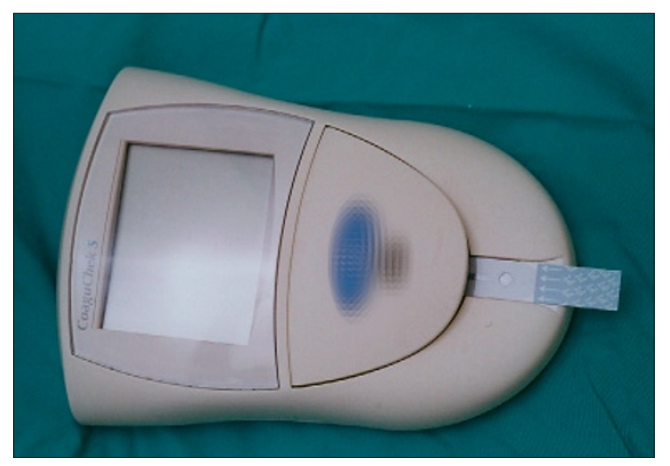

Fig. 5 The INR can be measured at the chair side by portable machines that require only a finger-prick blood sample

Current advice ${ }^{5}$ is that most surgical operations that can be performed in dental practice such as extractions and simple minor oral surgical procedures may be carried out if the INR is less than 3.0 without alteration of the warfarin dosage. If the INR is greater than 3.0 referral to the supervising physician is needed. If possible deep regional block anaesthesia should be avoided, for example by the use of intraligamentary injections in the mandible. Post-operative bleeding should be controlled by local measures such as suturing haemostatic packs.

Patients receiving heparin will not be encountered regularly in dental practice. Those who may seek treatment are patients on haemodialysis due to renal failure. These patients are heparinised on the days they are dialysed (normally three alternate days a week). However, due to the short half-life of heparin (around 5 hours) the effect is short lived and treatment can be performed safely on the days between dialysis.

\section{Choice of anaesthesia}

Anaemia should be corrected before intravenous sedation or general anaesthesia as the reduction in oxygen carrying capacity could be dangerous. 


\section{Drug interactions}

Many drugs prescribed by dentists interfere with warfarin. Some of these are important and can cause serious bleeding
The use of nitrous oxide sedation is probably best avoided in patients with pernicious anaemia, as one of the side effects of this gas is the production of vitamin $\mathrm{B}_{12}$ deficiency. ${ }^{2}$

The concern for patients with bleeding disorders is local anaesthesia. The use of deep injections such as inferior alveolar nerve blocks is contraindicated in patients with bleeding problems unless some form of prophylaxis has been provided. This is for fear of producing a bleed that may track around the pharynx leading to airway obstruction. Fortunately there are alternative methods of anaesthetising mandibular teeth, which allow this problem to be circumvented. Haemophiliac patients who had received intraligamentary anaesthesia for restorative dentistry without administration of Factor VIII recorded no complications related to haemorrhage or haematoma formation. ${ }^{6,7}$ Infiltration injections should not produce significant problems.

\section{Medication prescribed}

A number of drugs that dentists may prescribe can interfere with haemorrhage control and cause bleeding; the classic example is aspirin, which interferes with platelet function as described above. In addition, some drugs commonly used in dentistry including analgesics and antimicrobials interact with anticoagulants. Aspirin, diclofenac, diflunisal, ibuprofen and prolonged use of paracetamol all increase the effect of warfarin. Penicillins can increase the prothrombin time when given to patients receiving warfarin. However this effect is uncommon. Erythromycin enhances the anticoagulant effects of both warfarin and nicoumalone by reducing the metabolism of the latter drugs. Combined use is not absolutely contraindicated but monitoring of the patient is required. The effect of warfarin is significantly increased by metronidazole due to the antibiotic inhibiting the metabolism of the anticoagulant. This interaction is clinically important. ${ }^{8}$ If metronidazole is essential then the dose of warfarin may have to be reduced. Tetracycline may enhance the anticoagulant effect of warfarin and the other coumarin anticoagulants. Miconazole enhances the anticoagulant effect of warfarin even after topical use. Dentists should know about this important interaction as it may lead to catastrophic bleeding. A case has been reported of a warfarinised patient's INR increasing from 2.5 to 17.9 following the use of miconazole oral gel. ${ }^{9}$ One other drug worth mentioning is carbamazepine. This may reduce the effect of warfarin due to increased metabolism of the anticoagulant.

\section{Cross-infection control}

The chances of transmitting infected material between patients or from patient to operator should be minimised for all dental treatments. However, this is especially so when treating patients who are at high risk of carrying vira diseases such as HIV and hepatitis. Sadly some patients who have received treatments for bleeding disorders, principally multiple transfusions of blood products, fall into this category. Rigorous cross-infection control measures must be adopted when dealing with these patients.

\section{CONCLUSIONS}

Haematological problems impact on all aspects of dentistry. The taking of a thorough history and close liaison with medical colleagues will help reduce the problems that may occur in the patient with disorders of the blood. Patients with clotting defects represent a challenge to both surgical and restorative dentistry. Although al patients should have their management discussed with their supervising haematologist or physician when surgical procedures are to be performed, many cases can be adequately treated using local measures and skills commonly employed by dentists.

1. Seymour R A, Meechan J G, Walton J G. Adverse Drug Reactions in Dentistry. 2nd ed. 140-142. Oxford: Oxford University Press, 1996.

2. Donaldson D. Meechan J G. The hazards of chronic exposure to nitrous oxide. An update. BrDent J 1995; 178: 95-100.

3. Peterson L J. Antibiotic prophylaxis against wound infections in oral and maxillofacial surgery. J Oral Maxillofac Surg 1990 48: 617-620.

4. Wahl M J. Myths of dental surgery in patients receiving anticoagulant therapy. J Am Dent Assoc 2000; 131: 77-81.

5. Dental Practitioners' Formulary 2002-2004. London: BDA, BMA, RPSGB. ppD8, 117-119.

6. Ah Pin PJ. The use of intraligamental injections in haemophiliacs. Br Dent J1987: 162: 151-152.

7. Spuller R L. Use of the periodontal ligamentary injection in dental care of the patient with haemophilia - a clinical evaluation. Spec Care Dent 1988; 8: 28-29.

8. Kazmier FJ. A significant interaction between metronidazole and warfarin. Mayo Clin Proc 1987; 51: 782-784.

9. Colquhoun M C, Daly M, Stewart P. Beeley L Interaction between warfarin and miconazole oral gel. Lancet 1987; i: 695-696. 